\title{
A versatile apparatus for on-line emission channeling experiments
}

\author{
M. R. Silva, ${ }^{1}$ U. Wahl, ${ }^{1,2}$ J. G. Correia, ${ }^{1,2}$ L. M. Amorim, ${ }^{3}$ and L. M. C. Pereira ${ }^{3}$ \\ ${ }^{1}$ Centro de Física Nuclear, Universidade de Lisboa, 1649-003 Lisboa, Portugal \\ ${ }^{2}$ Unidade de Física e Aceleradores, IST/ITN, Instituto Superior Técnico, Universidade Técnica de Lisboa, \\ 2686-953 Sacavém, Portugal \\ ${ }^{3}$ Instituut voor Kern- en Stralingsfysica, KU, Leuven, 3001 Leuven, Belgium
}

(Received 29 April 2013; accepted 24 June 2013; published online 18 July 2013)

The concept and functionality of an apparatus dedicated to emission channeling experiments using short-lived isotopes on-line at ISOLDE/CERN is described. The setup is assembled in two functional blocks - (a) base stand including beam collimation, implantation and measurement chamber, cryogenic extension, and vacuum control system and (b) Panmure goniometer extension including maneuvering cradle and sample heating furnace. This setup allows for in situ implantation and sample analysis in the as-implanted state and upon cooling down to $50 \mathrm{~K}$ and during annealing up to $1200 \mathrm{~K}$. The functionality of the setup will be illustrated with the example of establishing the lattice location of ${ }^{56} \mathrm{Mn}$ probes implanted into GaAs. () 2013 AIP Publishing LLC. [http://dx.doi.org/10.1063/1.4813266]

\section{INTRODUCTION}

Emission channelling (EC) with position-sensitive detectors is a well-established technique for studying the lattice location of radioactive impurities implanted into single crystals. ${ }^{1-5}$ In the case of electron emitting radioisotopes, however, due to count rate level and noise related limitations of the detection systems, the technique was for long time restricted to isotopes with half-lives above $6 \mathrm{~h}$, with an effective count rate up to 200 events/s. ${ }^{6,7}$ On the other hand, modern radioactive ion beam facilities such as ISOLDE/CERN ${ }^{8-11}$ can deliver intense beams of short-lived isotopes.

With the recent implementation of fast, self-triggered $\mathrm{Si}$ pad detectors for emission channeling, ${ }^{12}$ high count rates up to 4000 events/s became achievable and a large variety of short-lived probe isotopes can now be used in EC experiments at the ISOLDE facility.

This paper details the functional concept of a specially developed experimental chamber mounted on-line at the ISOLDE beam lines, thus allowing to implant radioactive probe isotopes while simultaneously measuring the electron EC angular anisotropy patterns. To guarantee the $\sim 0.1^{\circ}$ of angular resolution regarding the $\pm 2.5^{\circ}$ range of analysis at the electron detector, the beam spot should not exceed $1 \mathrm{~mm}$ diameter and always hit the sample at the same place.

The critical task of this setup is to provide the precise beam spot while coping with the variety of emittances, yields and beam line focusing parameters of the many radioactive beams at ISOLDE. Additionally, the full setup should be movable and self-sustained to be easily coupled, in a reproducible way, to different beam lines at different locations of the radioisotope separator, adapting itself to the multi-user multibeam annual schedule of the laboratory. Following the description of the experimental setup an example of on-line EC experimental results is shown describing the lattice site location of the short-lived ${ }^{56} \mathrm{Mn}$ in GaAs.

\section{TECHNICAL CONCEPT}

The concept of the setup and the choice of materials were ruled by the following requirements:

- Compactness: The setup should be compact and selfsustained to allow for easy coupling to different beam lines at ISOLDE and for easy shipping between different laboratories.

- Stability and reproducibility: The alignment of the different beam components of the chamber, i.e., ion beam collimation, beam implantation trajectory, and beam monitoring must be precise, stable, and easily checkable, assembled together when positioning the chamber at the beam line. In particularly, the sensitive alignment of the setup to the beam line must be reproducible and straightforward.

- Functionality: The mechanical assembly and access to the different functional blocks must be made easy to allow a fast intervention during beam times when relatively high levels of radiation might be present, e.g., when working with very short-lived radioisotopes, or to allow for the change of disposable contaminated collimators, which are a perturbing source of background radiation.

- Construction: The materials and construction must be compatible with operation in high vacuum (better than $1 \times 10^{-6}$ mbar) and present very low level of stray magnetic fields that could interfere with the trajectories of the emitted electrons and distort their angular distribution patterns.

\section{BLOCK DESCRIPTION}

\section{A. Base stand}

Figure 1 shows a schematic view of the EC assembled setup. In this stand can be distinguished three mechanical functional sections: (a) beam tube and collimation chamber, 


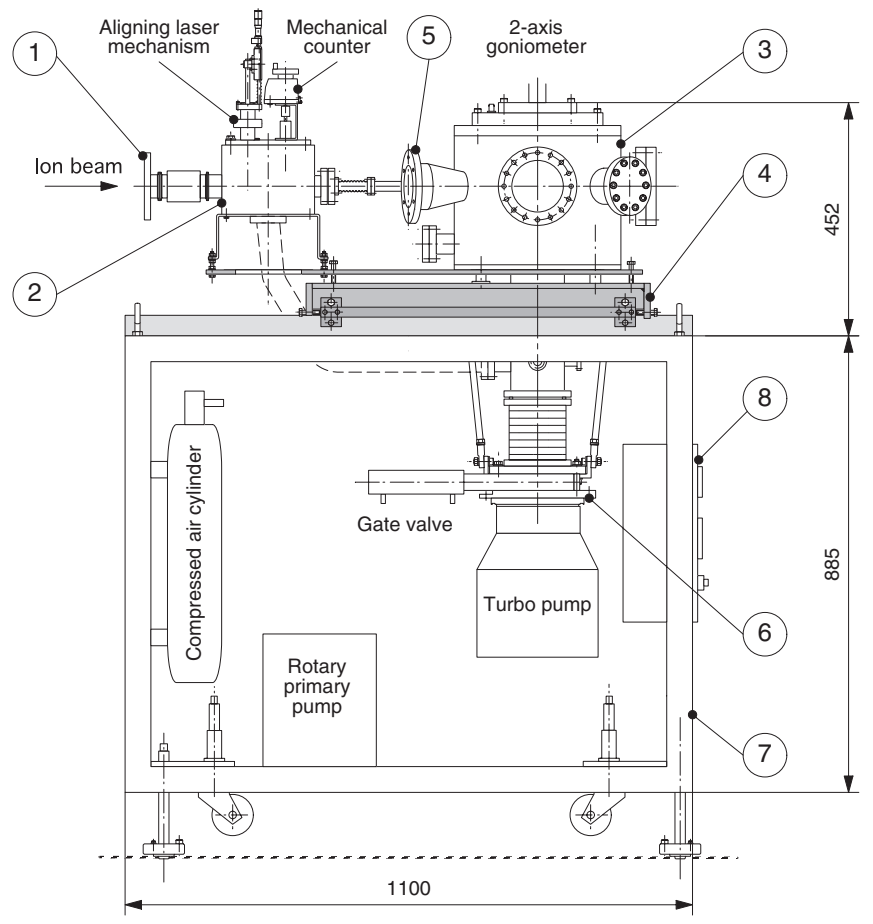

FIG. 1. Schematic view of the EC chamber assembly (dimensions in $\mathrm{mm}$ ): 1-ISOLDE beam-line ISO100 flange; 2-collimation block; includes: variable aperture collimator, lead shield, and laser alignment device; 3-sample implantation, annealing and measurement experimental chamber; 4-XZY sliding orientation cradle; 5-detector flanges DF30 and DF17; 6-vacuum block; 7-movable supporting frame stand with wheels and adjustable supporting pads; and 8-auxiliary control equipment panel. (b) experimental, and (c) vacuum block, all mounted on a supporting movable cradle.

The whole setup is assembled together on the top of a fully adjustable frame structure conceived to support weights up to $400 \mathrm{~kg}$. This frame, Figure 1(7), is fitted with wheels and vertically adjustable supporting pads according to the standard three-leg geometry for experiment locations at ISOLDE. With a few exceptions, the construction material chosen for the vacuum chamber and its support elements is nonmagnetic stainless steel AISI 304. With some exceptions, all flanges and fittings are standard CF type with copper seals. Flat Viton seals are optionally used on flanges that need to be frequently opened for alignment purposes or sample manipulations.

Figures 2 and 3 show the respective vertical and horizontal cross-section views of the assembled EC chamber.

\section{Implantation and sample measurement chamber}

In this chamber samples are implanted, optionally annealed, or cooled and the actual emission channeling measurements take place. This chamber consists of a block of cylindrical shape (D: $315 \mathrm{~mm}, \mathrm{H}: 260 \mathrm{~mm}$, internal) shown in Figures 1-3.

Several CF flanges of various diameters are inserted along its perimeter. Auxiliary devices such as cryogenic cooler, Faraday cup, view ports, and load lock are mounted on these flanges. There are also two special format flanges (DF17 and DF30, Figures 2(2) and 3(4), reserved to mount electron detector cases at angles of $17^{\circ}$ and $30^{\circ}$, respectively, with the beam line. On the bottom of the sample chamber,

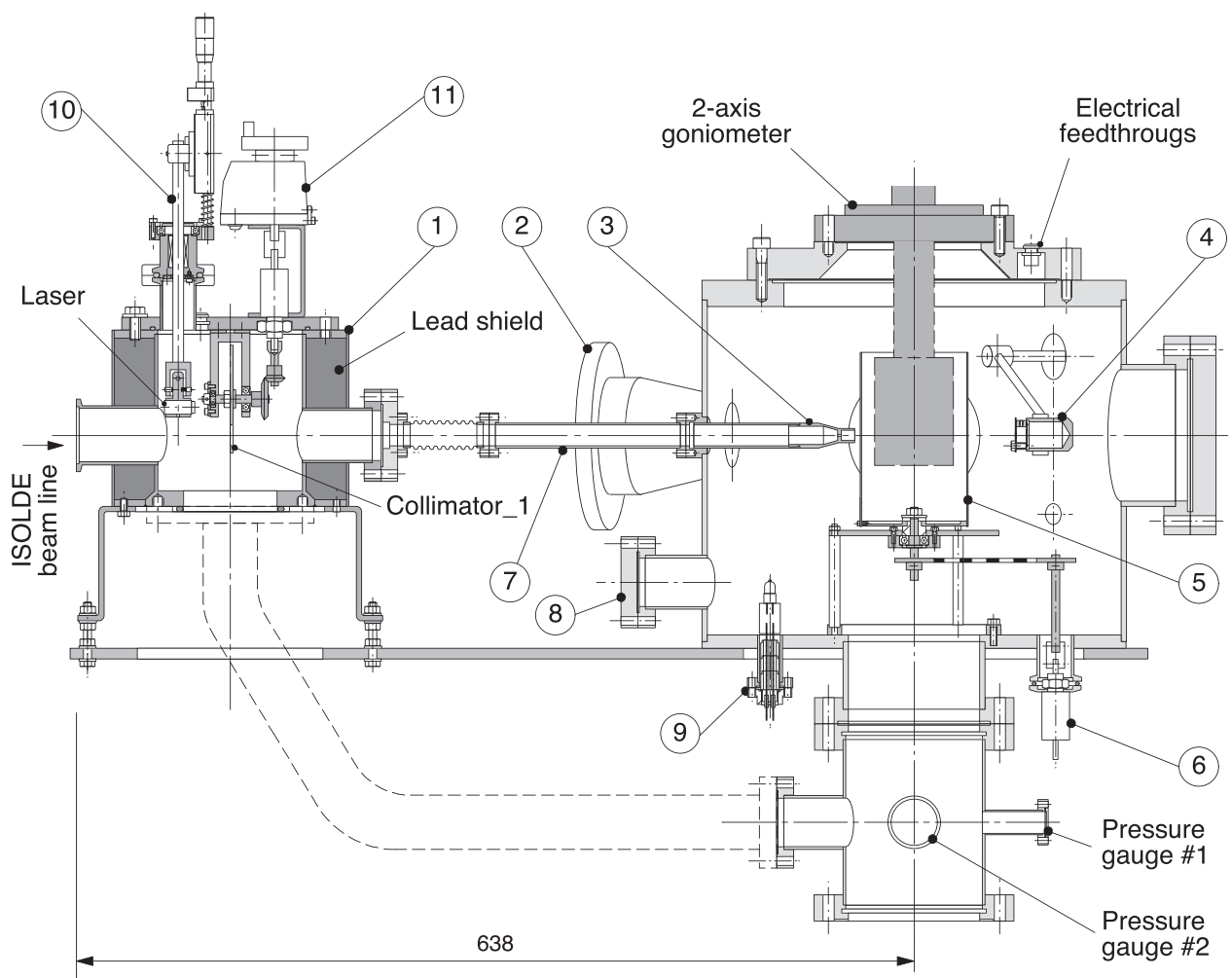

FIG. 2. EC setup vertical cross section: 1-collimator chamber with lead shield, rotatable disk with collimator holes, alignment laser; 2-detector flange DF17; 3-fixed aperture removable collimator; 4-movable Faraday cup; 5-revolving thermal shield; 6-thermal shield actuator; 7-ion beam transport tube; 8-spare flange; 9-halogen lamp; 10—alignment laser mechanism; and 11—mechanical counter. 


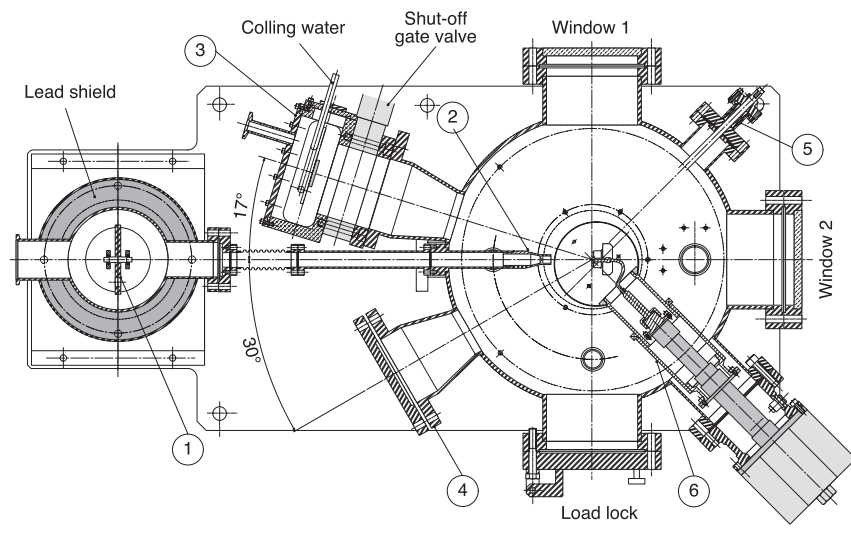

FIG. 3. EC chamber horizontal cross section: 1-lead shield and rotatable disk with collimator holes; 2 -fixed aperture removable collimator; 3 -fast Si pad $\left(28 \times 28 \mathrm{~mm}^{2}\right)$ electron detector block mounted on the DF17 flange; 4-spare detector flange DF30; 5-displaceable Faraday cup mechanism; and 6-cryogenic block, cryogenic shields, and cold finger.

bolted to a CF100 flange, protrudes a manifold linked to the vacuum station through a gate valve and with CF25 ports for vacuum measurement and control. Two other CF25 flanges are inserted in the chamber bottom, one for the inside lightening quartz lamp, Figure 2(9), and the other for the thermal shield actuator feedthrough, Figure 2(6).

On the chamber top flange is located the CF100 flange where two different goniometers can be mounted: either a 2axis assembly (HPT-RX VG Scienta) or a high precision 3axis assembly ("Slimline" from Panmure Instruments Ltd.). Both goniometers have sample holders equipped with resistive heating stages for sample heating up to $1200 \mathrm{~K}$. The heating stage for the Panmure goniometer is a non-standard piece that is described in more detail in Sec. III A 3. Both goniometers are also equipped with X,Y,Z-translation stages which assure that the sample can be properly aligned with respect to the beam and also retracted upwards for implantation current measurement in the Faraday cup.

During the annealings at high temperature (up to $1200 \mathrm{~K}$ ) detectors and other devices inside the chamber must be eventually thermally protected. This is accomplished by placing in the chamber a revolving thermal shield, Figure 2(5), controlled through an external actuator, Figure 2(6).

At $90^{\circ}$ from each other there are three CF100 flanges with viewports in two of them, Figure 3, and a load lock in the third one for sample changing and inside manipulations.

In Figure 4 the Faraday cup with a 3-electrode structure is shown: Faraday cup, collimator, and secondary electron suppression ring. This compact assembly, which is mounted behind the sample, can be moved in or out of the beam axis with the help of an actuator with rotation and translation degrees of freedom, Figure 4(4).

\section{Beam tube and collimation}

The 30-60 keV ISOLDE ion beams have varying emittances, depending on the type of target/ion source, e.g., plasma, surface, or laser ionization. Additionally, the optimum focusing at the sample depends on the particular beam line used and on the experimental setup. It is hence impor-

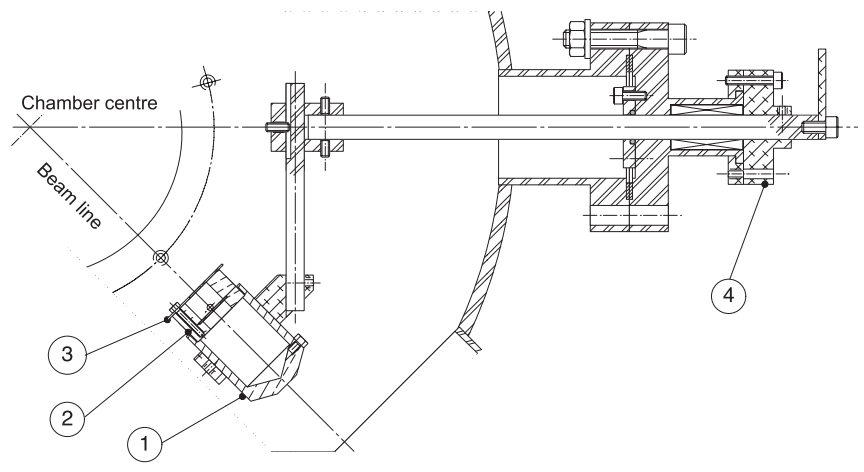

FIG. 4. Faraday cup: 1-Faraday cup; 2-suppressor ring; 3-shield plate; and 4-Faraday alignment mechanism (translation and rotation).

tant to have a precise and reliable beam transport geometry from the ISOLDE beam line end flange to the sample. This is achieved by a set of two rigidly aligned collimators (Figures 2(1), 3(1) and 2(3), 3(2)), which are mounted $520 \mathrm{~mm}$ apart and connected by a beam transport tube.

Figure 5 shows the 1 st beam forming collimator consisting of an externally actuated rotatable stainless steel disk,

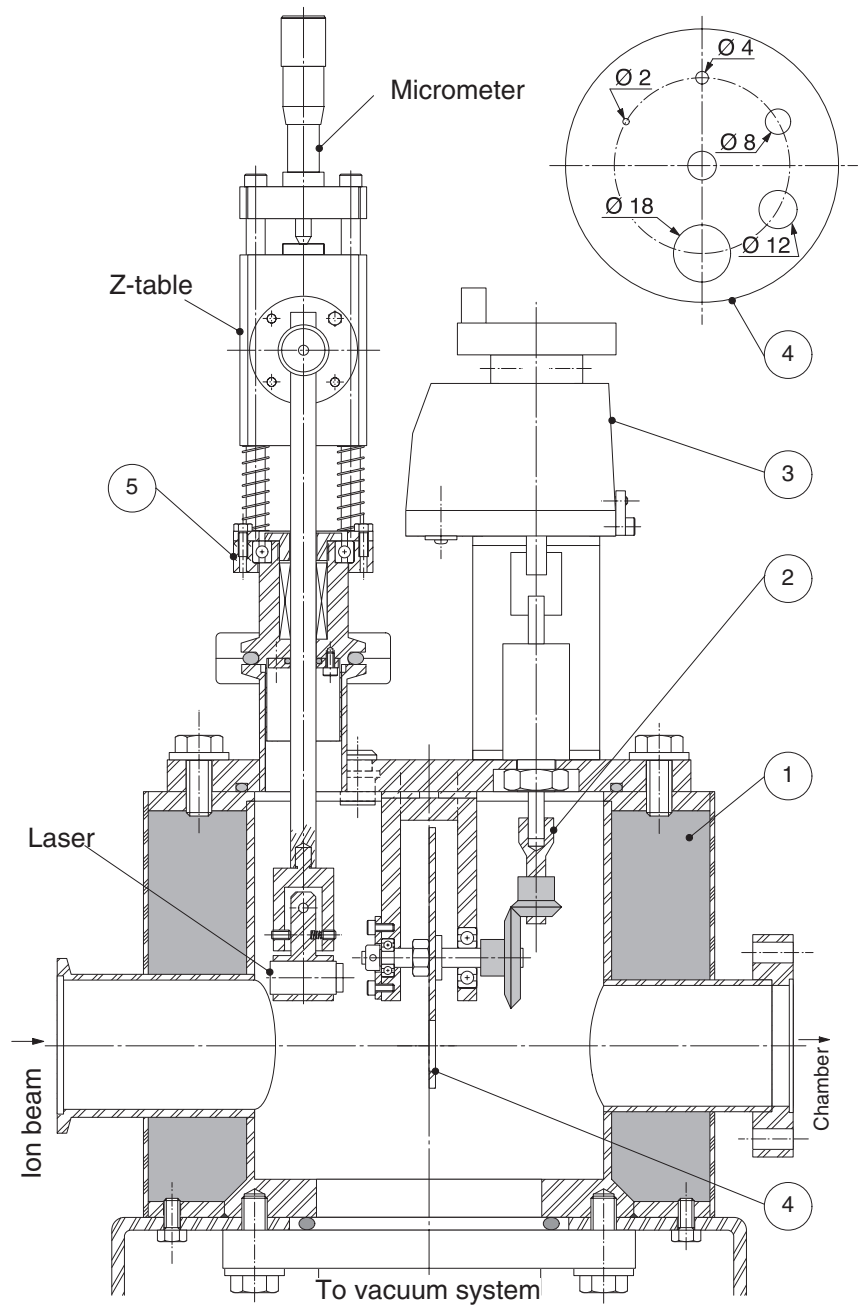

FIG. 5. Beam forming collimator and laser alignment mechanism: 1collimator chamber and lead shield; 2-beam forming diaphragm mechanism; 3-counter for positioning the variable diaphragm; 4-rotatable disk with collimator holes of various diameters; and 5-laser alignment mechanism. 
supported in ball bearings, with a blank sector and 5 round holes of different diameters on it $(2,4,8,12$, and $18 \mathrm{~mm})$. The disk is electrically isolated from ground allowing for measurement of the impinging and retained beam current (although without secondary electron emission suppression), which is usually performed during beam tuning with non-radioactive beams. The position of the rotatable collimator disk is manually controlled and read out in an external mechanical counter, located on the top flange of the collimator chamber. To guarantee the exact positioning of the disk, without backlash from outside the vacuum, a special rotation slack-free feedthrough from "Ferrofluidics" (vacuum parameters pressure $1 \times 10^{-9}$ mbar and leakage rate $\left.1 \times 10^{-11} \mathrm{mbar} 1 / \mathrm{s}\right)$ is used.

The collimator chamber consists of an internal vacuumtight cylinder (D: $110 \mathrm{~mm}, \mathrm{H}: 122 \mathrm{~mm}$ ) surrounded by a thin external wall (D: $170 \mathrm{~mm}, \mathrm{H}: 122 \mathrm{~mm}$ ) with the space between walls filled up with $1 \mathrm{~mm}$ diameter lead balls to shield $\gamma$-radiation from the contaminated 1 st impact collimator. The chamber bottom flange can be connected to an independent vacuum pump or directly to the main pumping system. In the collimator chamber is also mounted on the laser alignment system, Figure 5(5). This system consists of a sharp focus solid-state laser (100 $\mathrm{mW}$, red) suspended from a vertical positioning shaft coupled to a precision manipulator mounted on the top of the chamber. Besides the fine adjustment in height, this manipulator moves the laser in and out of the "in-beam" position. To properly orient the laser beam the system has two more degrees of freedom: rotation on the XY plane and tilt of the laser head on the XZ plane. When in the "in-beam" position, the laser is collinear with the ion-beam axis, along which are aligned both collimators, to allow for an easy and fast localization of the beam spot on the sample. Monitoring the position of the laser beam that is reflected from a mounted sample with mirror-finish surface also allows for easy calibration and control of the angular positions of the goniometer that rotates the sample.

In Figure 6 a drawing of Collimator- 2 that faces the sample is shown. Besides its collimating function, the geometry of this part also decreases significantly the exposure of the detector to beta particles emitted by probe atoms that were stopped in the collimator. This special nozzle-shaped collimator is made of copper-beryllium alloy doubly plated with a $2 \mu \mathrm{m}$ silver inner layer covered by a $2 \mu \mathrm{m}$ gold layer (silver layer avoids copper in-diffusion of the gold layer). The use of $\mathrm{Cu}-\mathrm{Be}$ alloy and gold plating prevents this part from oxida-

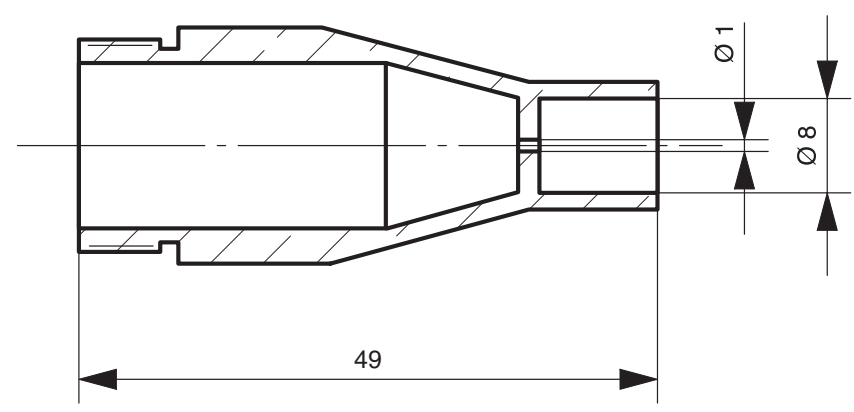

FIG. 6. Collimator-2 nozzle (defines the beam spot on the sample). tion and assures a smooth movement inside the stainless steel thread, which is essential for fast exchange of contaminated collimators with high radiation levels.

The $1 \mathrm{~mm}$ aperture fixed nozzle (alternatively 0.5 or $1.4 \mathrm{~mm}$ ) is located at $54 \mathrm{~mm}$ from the sample surface, at a distance that does not interfere with the detector line of sight and the goniometer head movements. Several units were made to allow for easy replacement during beam time, when high level contamination is attained or when different beam diameters are required.

The ion beam tube end section, Figure 2(7), where the nozzle is fixed has a rigorous, low tolerance, perpendicular alignment with both the chamber wall and the bottom surface following the theoretical beam axis, to guarantee optimum beam alignment. This mechanical precision was achieved by first welding a raw piece of tube on the chamber wall, with an unfinished CF25 flange already attached. Then the CF25 flange on one side and the fine screw thread at the tube end, inside the chamber, were machined with high precision as one piece.

\section{Non-standard sample holder heater}

The originally mounted VG goniometer on the EC setup has a resistive heater included in the sample holder, but the Panmure goniometer has not.

Thus a special sample holder plug with resistive heating was developed, Figure 7, to be inserted into the original Panmure sample holder tube, Figure 7(9). Two independent blocks compose this sample holder: the sample holder itself and a supporting base that includes the electrical feeds.

The central part of the sample holder block is a commercially available molybdenum button heater (HeatWave Labs Inc.), ${ }^{13}$ Figure $7(1)$, on which surface samples are mounted, secured by a molybdenum frontal plate, Figure 7(8). The temperature is measured by clamping a type $\mathrm{K}$ thermocouple underneath the frontal plate next to the sample. Two tantalum and stainless steel heat shields surround the heating element fixed in a ceramic base, Figures 7(2), 7(3), and 7(4). From this base protrude two electrical contacts, power and ground.

This entire sample holder block is removable allowing for sample mounting and maintenance outside the implantation

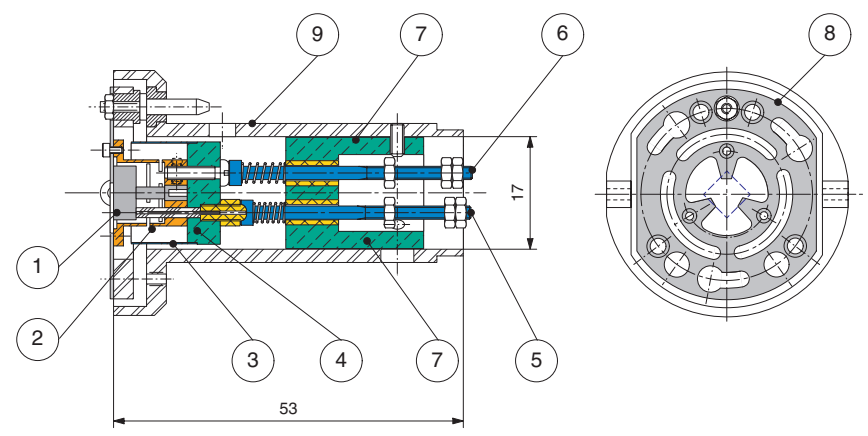

FIG. 7. Non-standard removable sample holder heater: 1-heating element; 2-tantalum thermal shield; 3-stainless steel thermal shield; 4-ceramic heater base; 5-power feed; 6-ground connection; 7-back support; 8molybdenum frontal holding plate; and 9-Panmure sample holder tube. 
chamber, without touching the aligned goniometer head. The fixed ceramic back support, Figure 7(7), includes two electrical feeds (Figures 7(5) and 7(6)) that, compressed by springs to the sample holder, power the button heater.

The overall construction of the heater is small and flexible enough as to not interfere with the goniometer head movements. It allows to reach annealing temperatures up to $1173 \mathrm{~K}$ while at the same time minimizing light emission. Nonetheless, when it is required to measure electron mission channeling patterns during annealing above $573 \mathrm{~K}$, a thin alumunized Mylar foil is mounted in front of the Si pad detector. Thus, the highest measurement temperature which can be reached before the detector responds to the light is $1073 \mathrm{~K}$.

\section{Cooling station}

Fixed to the side of the implantation chamber is the CTI Cryogenics cooling station, Figure 8(6). It consists of a $\mathrm{He}$ external compressor and a cryogenic head inserted into the chamber through a CF flange, Figure 8.

An electrical resistance heater wound around the cold finger second stage, Figure 8(2), and a diode temperature gauge attached to the sample holder are used to control the sample temperature. A second temperature measuring diode is attached to the sample holder, near the sample, to monitor its temperature.

The data from the control diode is introduced in a PID controller that fixes the working set point temperature.

To guarantee the freedom of movements of the goniometer head a flexible OFC copper braid, Figure 8(1), is fixed between the sample holder and the cold-finger second stage, closing the cold circuit.

Copper thermal shields protect the full thermal path between the sample holder and the cryostat (cold-finger, copper braid, cryostat stages). All the copper parts that make up this system are silver and gold plated to ensure the best thermal

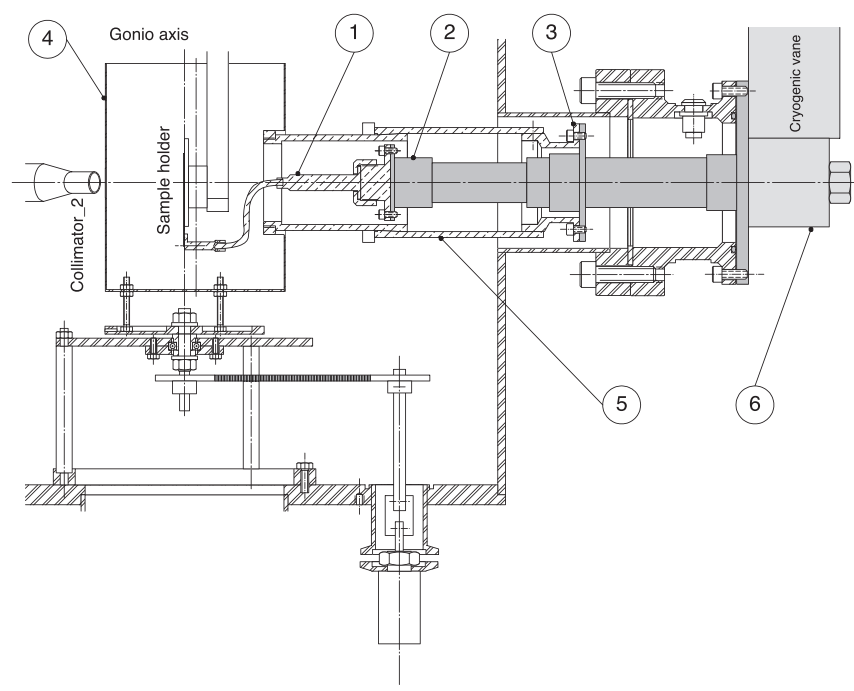

FIG. 8. Cryogenic block: 1 -cold finger and copper braid; 2 -cryostat 2nd stage $(4 \mathrm{~K}) ; 3$ - cryostat 1 st stage $(77 \mathrm{~K}) ; 4$-sample holder thermal shield; 5 - cold path thermal shield including sliding section; and 6-cryogenic vane connected to compressor. contact between dismountable parts and the cleanliness of the shields.

A section of the cryostat shield, Figure 8(5), extended over the thermal path between the central shield and the cryostat first stage can be displaced providing free access to the conduction path coupling for maintenance and mounting purposes.

\section{The sliding orientation cradle}

The full EC chamber assembly with the attached suspended turbo-pump block, Figure 1(6), sits on top of the sliding cradle, Figure 1(4). This setup permits a precise spatial adjustment of the whole chamber along the ISOLDE beam axis, once the assembly is set to the beam line.

This cradle, with the whole chamber attached, can be displaced along the top of the frame sliding on two longitudinal slide bars.

The fine positional adjustment of the chamber can be done using its 5 degrees of freedom fine adjustments: $X X$, YY, ZZ, 2 tilt motions (around XX and YY axis), and one rotation, around $\mathrm{ZZ}$ axis.

The heavy construction of the cradle allows the attenuation of vibrations, mainly from the vacuum block and an overall vibration free EC setup operation.

\section{Vacuum system and controls}

The vacuum system, Figure 1(6), is a standard turbo pump (500 1/min) and primary rough pump pair with oil mist filters and coupled to the implantation chamber through a gate valve pneumatically operated. Pneumatically operated gate valves also protect the electron detectors mounted in their special flanges, DF17/DF30. A standard Penning-Pirani gauge pair controls the vacuum quality and valve operation. The Penning gauge is located as far as possible from the detector positions and shielded with $\mu$-metal to avoid stray magnetic field interference with the measurements.

The normal operation vacuum level is around 1 $\times 10^{-6}$ mbar, but can reach $7 \times 10^{-7}$ mbar after prolonged pumping.

All the controls, displays, and interlock signaling lamps are located in a panel fixed on the front of the frame, Figure 1(8). In this board is also located the system operation set of interlocks. All needed low voltage power supplies as well as the compressed air cylinder for gate valve actuation are fixed on the assembly base frame, Figure 1(7). Alternatively, the compressed air can be drawn from the laboratory system through a pressure reducer.

\section{Panmure goniometer cradle}

The Panmure goniometer, when fully equipped for stepping motor operation, has a weight over $50 \mathrm{~kg}$. Thus a special supporting and manipulation cradle was built to allow for easy mounting and unmounting of the Panmure goniometer and at the same time maintain the compactness of the EC setup. Through this supporting cradle the weight on the 


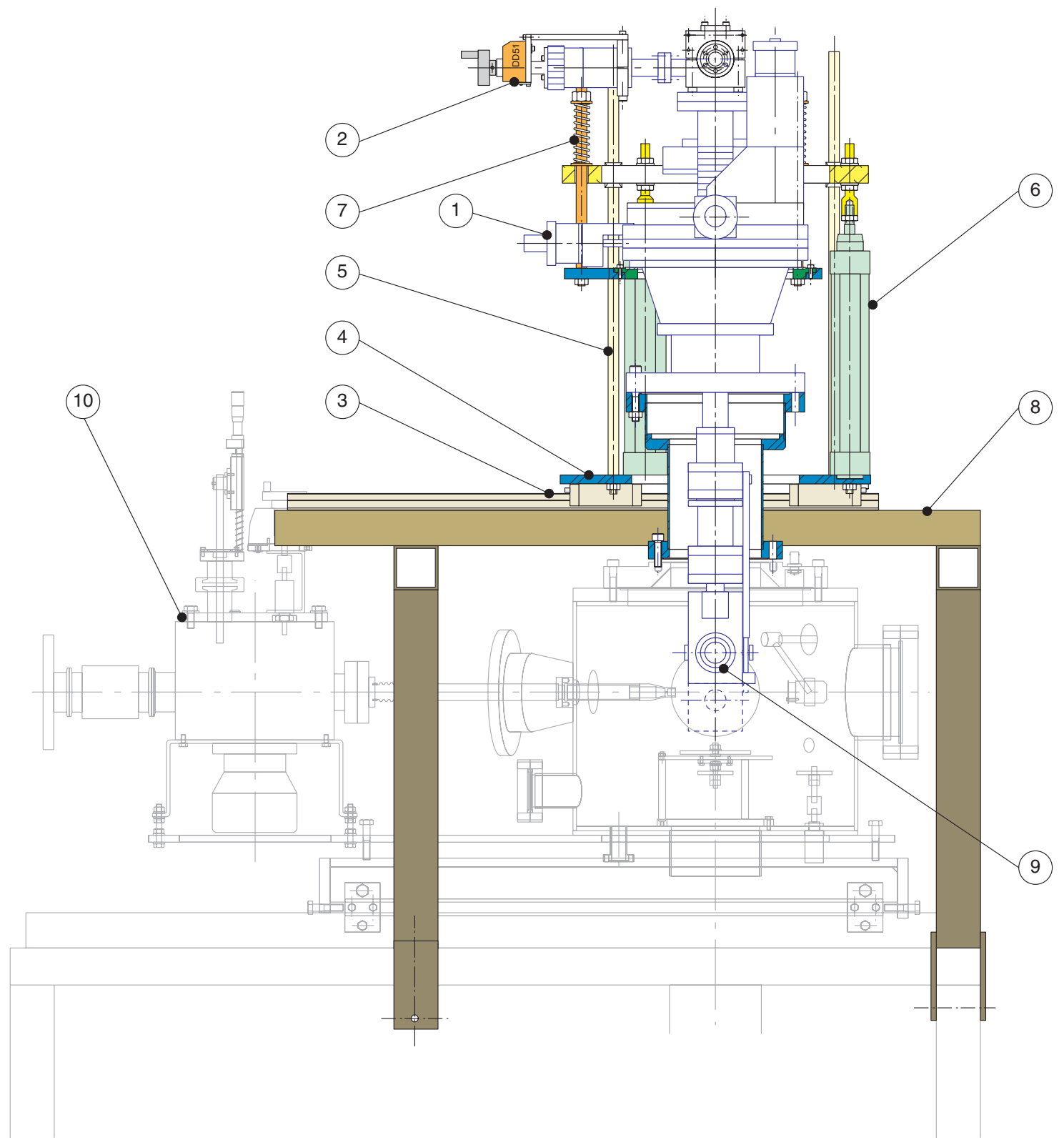

FIG. 9. Panmure goniometer stand: 1—goniometer; 2-goniometer mechanical actuators and counters; 3 -rail-slides set for transversal displacement; 4-lifting mechanism base; 5—vertical displacement guide; 6—pneumatic actuator; 7—heavy weight compensation springs; 8—fixed cradle; 9—goniometer sample holder; and 10-EC chamber and setup.

experimental chamber top is suppressed and servicing of the goniometer head is also eased for the experimentalist. This type of construction also still allows for the possible EC setup operation with the VG goniometer mounted by just lifting and shifting the Panmure goniometer along the cradle rails.

The Panmure goniometer stand consists of a stainless steel auxiliary cradle, Figure 9(8), that sits on the EC setup cradle top. This auxiliary cradle has a sliding table on top, Figures 9(3) and 9(4), allowing for the horizontal displacement of the goniometer and its stand.

The goniometer vertical displacement is obtained by a set of three pneumatic cylinders, Figure 9(6), and steered by guide bars, Figure $9(5)$.

The full weight of the goniometer is supported by a set of weight compensation springs, Figure 9(7), leaving only a residual weight of 3-4 $\mathrm{kg}$ to be exerted on the top of the experimental chamber when in use.

\section{RESULTS FROM EC ON-LINE EXPERIMENTS}

The EC setup is currently mounted at the GHM beam line of the ISOLDE facility. It has already been successfully used for a variety of experiments involving the lattice location of short-lived isotopes in semiconductors, e.g., for the radioactive probes ${ }^{11} \mathrm{Be}\left(\mathrm{t}_{1 / 2}=14 \mathrm{~s}\right),{ }^{24} \mathrm{Na}(15 \mathrm{~h}),{ }^{27} \mathrm{Mg}(9.5 \mathrm{~min}),{ }^{14}$ ${ }^{56} \mathrm{Mn}(2.6 \mathrm{~h}),{ }^{15-19}{ }^{57} \mathrm{Mn}(1.7 \mathrm{~min}),{ }^{61} \mathrm{Mn}(4.6 \mathrm{~s}),{ }^{61} \mathrm{Fe}(6 \mathrm{~min})$, ${ }^{61} \mathrm{Co}(1.6 \mathrm{~h}),{ }^{17}{ }^{65} \mathrm{Ni}(2.5 \mathrm{~h})$.

As an example we present some of the results of lattice location experiments of ${ }^{56} \mathrm{Mn}$ implanted into GaAs discussed in detail in Refs. 16 and 18. Mn-doped GaAs represents a diluted magnetic semiconductor system where the 
possible lattice locations of $\mathrm{Mn}$ are of particular interest since the maximum achievable Curie temperature depends on the ratio of $\mathrm{Mn}$ incorporated in substitutional Ga sites vs. interstitial Mn. For the purpose of this experiment, the shortlived ${ }^{56} \mathrm{Mn}$ isotope was implanted on-line at ISOLDE using the setup described above into single crystals of GaAs. The ISOLDE beam was collimated using the variable size collimator and the implantation nozzle with $1 \mathrm{~mm}$ diameter hole. Since the ISOLDE beams cannot be focused better than a few $\mathrm{mm}$ diameter by the beam line quadrupole focusing optics, typical transmission values for a $1 \mathrm{~mm}$ collimator are $\sim 20 \%-$ $30 \%$ for surface ionized beams and $\sim 30 \%-50 \%$ for beams produced by laser ionization which results in beams of sharper emittance (as in the case of $\mathrm{Mn}^{+}$).

The ${ }^{56} \mathrm{Mn}$ implantation energies were between 30 and $50 \mathrm{keV}$ and typical implantation currents around 30-100 pA. The beam current was reliably measured with proper suppression of secondary electrons before the actual implantations, using the Faraday cup described in Sec. III A 1. During the implantation, the current on the sample inside the goniometer (including emission of secondary electrons) was monitored and integrated using a pico-amperemeter. The collected charge, which was corrected using a scaling factor obtained from comparing current measurements in the Faraday cup and on the sample itself, allowed to determine reliable values of $\sim 2 \times 10^{13} \mathrm{~cm}^{-2}$ for the implantation fluence.

The implantations were performed at room temperature under a tilt angle of $17^{\circ}$, i.e., with the surface normal of the sample pointing towards the position-sensitive detector, which allowed direct measurement of the $\langle 100\rangle$ emission channeling pattern from the surface orientation during the implantation. When the implantation was completed, the crystal was rotated in order to measure the emission channeling patterns of the off-surface directions $\langle 111\rangle,\langle 110\rangle$, and $\langle 211\rangle$, each of the measurements taking about $10-15$ min with a detector count rate of several $\mathrm{kHz}$. Following the as-implanted measurements the sample was annealed for $10 \mathrm{~min}$ at $373 \mathrm{~K}$ under the chamber vacuum better than $1 \times 10^{-5}$ mbar, then allowed to cool down to room temperature and all emission channeling patterns remeasured.

This procedure was then repeated for a whole annealing sequence up to $973 \mathrm{~K}$ in steps of $100 \mathrm{~K}$. Typical radiation levels near the chamber during such an experiment are $\sim 10-50 \mu \mathrm{Sv} / \mathrm{h}$. In order to minimize the radiation exposure of the operating personnel the whole setup is placed behind a shielding of concrete blocks and only accessed during an experiment for short time periods when turning the goniometer.

The left hand column of Fig. 10, i.e., panels (a)-(d), shows the angular-dependent count rate of $B^{-}$particles emitted by ${ }^{56} \mathrm{Mn}$ in semi-isolating ( $\left.s i\right) \mathrm{GaAs}$ as a function of angle from the main crystallographic directions $\langle 100\rangle,\langle 111\rangle,\langle 110\rangle$, and $\langle 211\rangle$, following annealing at $373 \mathrm{~K}$. In order to quantitatively analyze the experimental patterns, these are fitted by a combination of simulated patterns for ${ }^{56} \mathrm{Mn}$ on different lattice sites inside the GaAs structure. The simulated patterns are calculated using the so-called "manybeam" approach, ${ }^{1,3}$ while the fitting routines used for that purpose have been described in detail in Refs. 3 and 6. The best fits are shown in the right hand column of Fig. 10, i.e., panels (e)-(h), and cor-

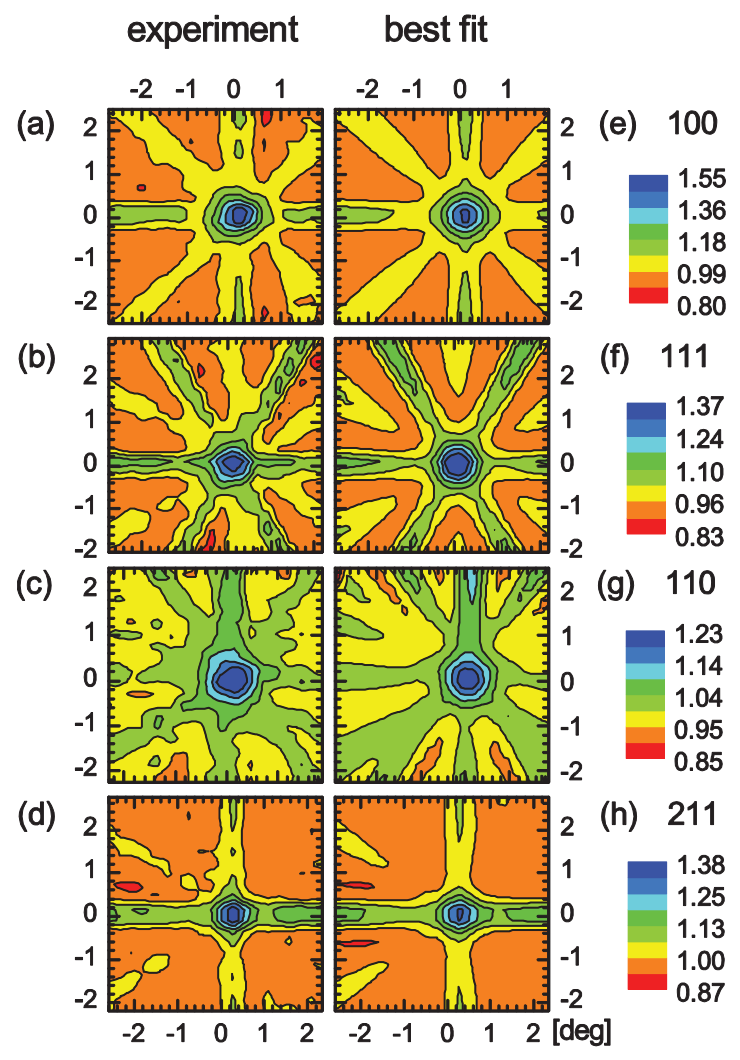

FIG. 10. (a)-(d) Normalized experimental $\beta^{-}$emission channeling patterns measured in the vicinity of the $\langle 100\rangle,\langle 111\rangle,\langle 110\rangle$, and $\langle 211\rangle$ directions of an (si)-GaAs single crystal implanted with ${ }^{56} \mathrm{Mn}$ following annealing at $100{ }^{\circ} \mathrm{C}$. (e)-(h) Corresponding best fits of simulated patterns obtained for the combination of $70 \%$ of the $\mathrm{Mn}$ atoms on $\mathrm{S}_{\mathrm{Ga}}$ sites and $28 \%$ on $\mathrm{T}_{\mathrm{As}}$ sites, respectively.

respond to a combination of $70 \%$ of ${ }^{56} \mathrm{Mn}$ atoms on substitutional $\mathrm{Ga}$ sites $\left(\mathrm{S}_{\mathrm{Ga}}\right)$ and $28 \%$ tetrahedral interstitial sites with nearest As neighbors $\left(\mathrm{T}_{\mathrm{As}}\right)$. The outcome of the annealing sequence was that following annealing at $873 \mathrm{~K}$ the interstitial Mn fraction was completely converted to substitutional Mn on $\mathrm{S}_{\mathrm{Ga}}$. The experiments hence gave direct evidence, that, in addition to the majority substituting for $\mathrm{Ga}$, a significant fraction of Mn occupies tetrahedral interstitial sites in one specific sublattice, and that an activation energy of $1.7-2.3 \mathrm{eV}$ is needed to convert this interstial fraction to substitutional.

\section{SUMMARY AND FURTHER DEVELOPMENTS}

In summary, we designed, constructed, and commissioned a compact stand-alone chamber for on-line and offline emission channelling measurements that combines ease of use with the possibility of in situ measurement of shortlived radioisotopes. The chamber can be equipped with precision goniometers, 3 or 2 axis, that allow implantation and sample orientation in a vacuum of $1 \times 10^{-6}$ mbar and thermal treatment of the samples at temperatures ranging from $50 \mathrm{~K}$ to $1200 \mathrm{~K}$. The chamber has already been successfully used in a variety of EC experiments with short-lived isotopes and it is expected that its field of applications will be further expanded. In that respect the following development work is in progress or foreseen: 
-Designing and commissioning of a second positionsensitive detector. In contrast with the $22 \times 22$ channels $\left(32 \times 32 \mathrm{~mm}^{2}\right) \mathrm{Si}$ pad detector currently in use, this Medipix type detector will be highly segmented (512 $\times 512$ pixels). This detector will work in parallel with the presently installed fast Si pad detector.

-Remote-controlled automation of the goniometer operation enabling efficient use of the chamber in particular with short-lived radioisotopes and allowing further reduction of the already quite low radiation exposure of the experimentalists.

\section{ACKNOWLEDGMENTS}

This work has been supported by the Portuguese Science Foundation FCT (projects CERN-FP-1235852011, CERN-FP-116320-2010, CERN-FP-109272-2009, CERN-FP-83506-2008, POCI-FP-81921-2007, and PDCTFP-63911-2005). The authors gratefully acknowledge the invaluable contribution of M. Cabaça and F. Baptista from ITN/IST-UTL, Sacavém, Portugal for their constant availability and the great quality of the machining work, welding and assembling of parts of this chamber. We thank all members of the Emission Channeling with Short-Lived Isotopes (EC-SLI) collaboration that have contributed by means of experimental results towards successful commissioning of the setup, in particular S. Decoster, D. J. Silva, E. Bosne, A. Costa, and V. Augustyns. We further acknowledge P. Weilhammer, E. Chesi, A. C. Marques, and C. Lacasta for the fast PAD detector and readout software developments without what EC experiments running short lives isotopes would not be possible.

${ }^{1}$ H. Hofsäss and G. Lindner, "Emission channelling and blocking," Phys. Rep. 201, 121 (1991).

${ }^{2} \mathrm{U}$. Wahl, "Emission channeling studies of Li in semiconductors," Phys. Rep. 280, 145 (1997).
${ }^{3} \mathrm{U}$. Wahl, "Advances in electron emission channeling measurements in semiconductors," Hyperfine Interact. 129, 349 (2000).

${ }^{4} \mathrm{U}$. Wahl, "Recent emission channeling studies in wide band gap semiconductors," Hyperfine Interact. 159, 363 (2004).

${ }^{5}$ A. Vantomme, B. De Vries, and U. Wahl, "Lattice location of RE impurities in III-nitrides,” Top. Appl. Phys. 124, 55 (2010).

${ }^{6}$ U. Wahl, J. G. Correia, A. Czermak, S. G. Jahn, P. Jalocha, J. G. Marques, A. Rudge, F. Schopper, J. C. Soares, A. Vantomme, P. Weilhammer, and the ISOLDE collaboration, "Position-sensitive Si pad detectors for electron emission channelling experiments," Nucl. Instrum. Methods Phys. Res. A 524, 245 (2004).

${ }^{7}$ A. C. Marques, U. Wahl, J. G. Correia, M. R. Silva, A. Rudge, P. Weilhammer, J. C. Soares, and the ISOLDE collaboration, "Noise and trigger efficiency characterization of cooled Silicon pad detectors," Nucl. Instrum. Methods Phys. Res. A 572, 1056-1064 (2007).

${ }^{8}$ E. Kugler, "The ISOLDE facility," Hyperfine Interact. 129, 23 (2000).

${ }^{9}$ U. Köster, "ISOLDE target and ion source chemistry," Radiochim. Acta 89, 749 (2001).

${ }^{10}$ L. Fraile, "Recent highlights from ISOLDE@CERN," Eur. Phys. J. A 25, 723 (2005).

${ }^{11}$ J. G. Correia, U. Wahl, and K. Johnston, "Nuclear radioactive techniques applied to materials research," Radiochim. Acta 100, 127 (2012).

${ }^{12}$ A. C. Marques, "Advanced Si-pad detector development and $\mathrm{SrTiO}_{3}$ studies by emission channelling and hyperfine interaction experiments," $\mathrm{Ph} . \mathrm{D}$. thesis (Faculdade de Ciências da Universidade de Lisboa, Portugal, 2009).

${ }^{13}$ HeatWave Labs Inc. USA, 195 Aviation Way, Watsonville, CA 95076, USA.

${ }^{14}$ L. M. Amorim, L. M. C. Pereira, S. Decoster, D. J. Silva, J. G. Correia, K. Temst, and A. Vantomme, "Direct measurement of the lattice location of Mg implanted in AlN," Appl. Phys. Lett. (submitted).

${ }^{15}$ S. Decoster, S. Cottenier, U. Wahl, J. G. Correia, L. M. C. Pereira, M. R. Da Silva, and A. Vantomme, "Diluted manganese on the bond-centered site in germanium," Appl. Phys. Lett. 97, 151914 (2010).

${ }^{16}$ L. M. C. Pereira, U. Wahl, J. G. Correia, S. Decoster, M. R. da Silva, J. P. Araújo, and A. Vantomme, "Direct identification of interstitial Mn in heavily $p$-type doped GaAs and evidence of its high thermal stability," Appl. Phys. Lett. 98, 201905 (2011).

${ }^{17}$ L. M. C. Pereira, U. Wahl, S. Decoster, J. G. Correia, L. M. Amorim, M. R. da Silva, J. P. Araújo, and A. Vantomme, "Mixed Zn and O substitution of Co and Mn in ZnO," Phys. Rev. B 84, 125204 (2011).

${ }^{18}$ L. M. C. Pereira, U. Wahl, S. Decoster, J. G. Correia, M. R. da Silva, A. Vantomme, and J. P. Araújo, "Stability and diffusion of interstitial and substitutional Mn in GaAs of different doping types," Phys. Rev. B 86, 125206 (2012).

${ }^{19}$ L. M. C. Pereira, U. Wahl, S. Decoster, J. G. Correia, M. R. da Silva, A. Vantomme, and J. P. Araújo, "Evidence of N substitution by Mn in wurtzite GaN," Phys. Rev. B 86, 195202 (2012). 